\title{
Research Paper \\ Relationship Between Visual, Hearing and Memory Disabilities and Hand Grip Strength and the Systems Usability Available to the Elderly Living in Nursing Homes in Kerman, Iran
}

\author{
Vafa Feyzi $^{1,2},{ }^{*}$ Naser Hasheminejad ${ }^{1}$, Akram Sadat Jafari Roodbandi $^{3}$
}

1. Department of Occupational Health, School of Public Health, Kerman University of Medical Sciences, Kerman, Iran.

2. Health Center, Saghez Branch, Kurdistan University of Medical Sciences, Sanandaj, Iran.

3. Department of Occupational Health, School of Public Health, Bam University of Medical Sciences, Bam, Iran.

\begin{tabular}{|c|c|}
\hline $\begin{array}{l}\text { Use your device to scan } \\
\text { and read the article online }\end{array}$ & Cltation: Feyzi V, Hasheminejad N, Jafari Roodbandi AS. [Relationship Between Visual, Hearing and Memory Disabilities \\
\hline 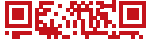 & and Hand Grip Strength and the Systems Usability Available to the Elderly Living in Nursing Homes in Kerman, Iran (Persian)]. \\
\hline patents & Iranian Journal of Ageing. 2017; 11(4):484-493. http://dx.doi.org/10.21859/sija-1104484 \\
\hline 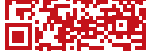 & dol: http://dx.doi.org/10.21859/sija-1104484 \\
\hline
\end{tabular}

Received: 06 Jul. 2016 Accepted: 18 Oct. 2016

Keywords: System usability, Disabilities, Hand-Grip strength, Elderly, Nursing homes

\section{ABSTRACT}

Objectives As people get older, their body systems become disrupted. As the elderly lose their abilities, they become more dependent on others. This study aimed to investigate the relationship between visual, hearing, and memory disabilities as well as hand grip strength and the systems usability available to the elderly living in nursing homes in Kerman, Iran.

Methods \& Materials This cross-sectional study was conducted on 110 elderly participants (58 men and 52 women) living in nursing homes in Kerman. To investigate hearing, memory, and visual disabilities, the audiometry method, standard memory assessment questionnaire and E chart were utilized, respectively. Jamar Dynamometer was used to measure hand grip strengths. The systems usability (television, radio, and refrigerator) was measured via System Usability Scale (SUS) and analyzed by statistical tests with 0.05 level of significance.

Results The average age of the participants was 75.35 years. Hearing and visual disabilities were significantly different in left ears and eyes. There was no significant difference between men and women regarding their memory disability. However, hand grip strengths were significantly different in men and women $(P=0.0001)$. The highest usability for both men and women groups was in using the refrigerator and the lowest in using television. The mentioned disabilities and hand grip strength of the elderly had a significant relationship with the ability to use television, refrigerator, and radio.

Conclusion With increasing age, the disabilities of the elderly get intensified and their ability to use systems decrease. Thus, it is necessary to prevent their loss of abilities in order to enhance their systems usability.

\section{* Corresponding Author:}

Naser Hasheminejad, PhD

Address: Department of Occupational Health, School of Public Health, Kerman University of Medical Sciences, Haft Bagh Alavi Blvd., Kerman, Iran. Tel: +98 (34) 31325111

E-mail: n_hasheminejad@kmu.ac.ir 


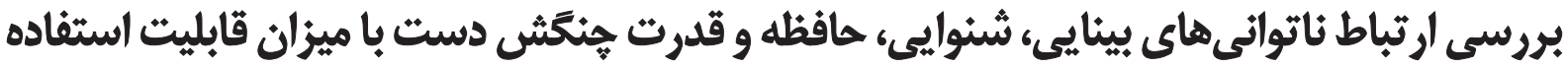

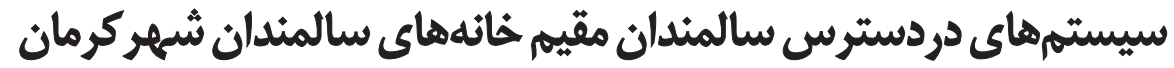

\author{
وفا فيضى "ما، "ناصر هاشمىنزاد'، اكرمسادات جعفرىرودبندى"

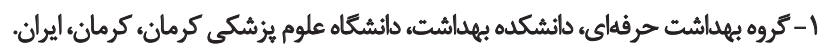

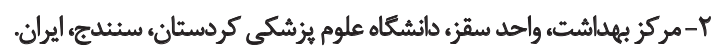

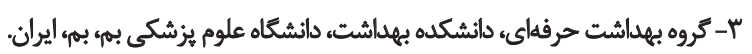

\begin{abstract}
حكبد

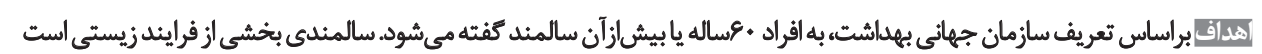

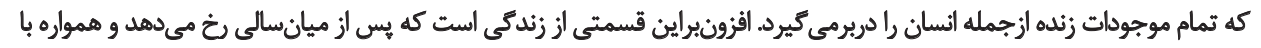

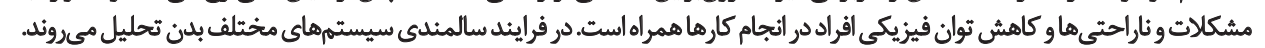

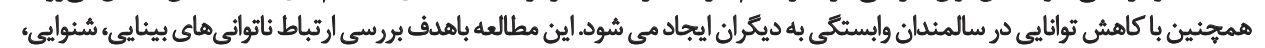

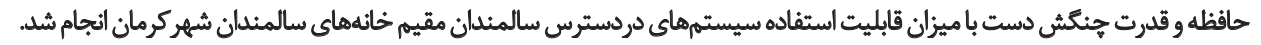

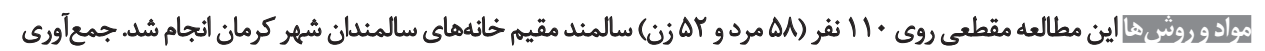

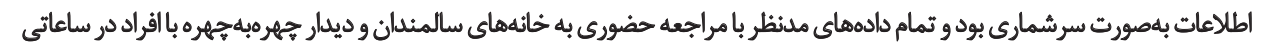

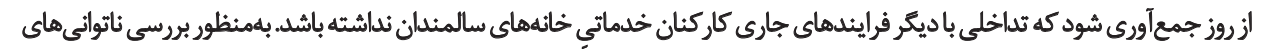

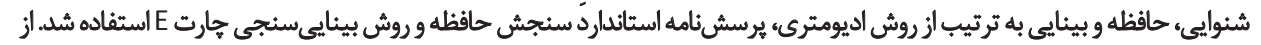

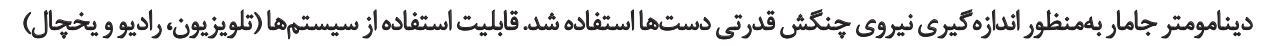

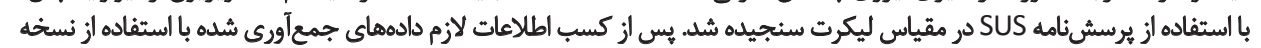

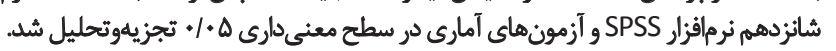

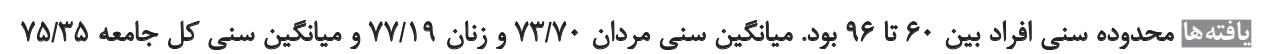

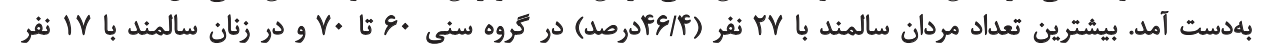

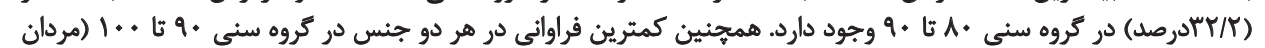

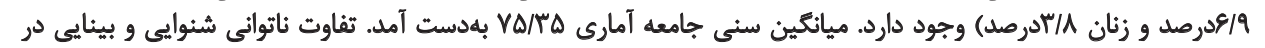

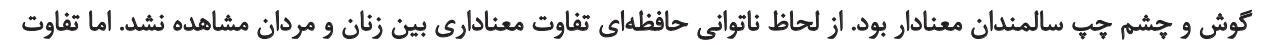

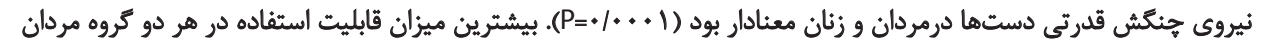

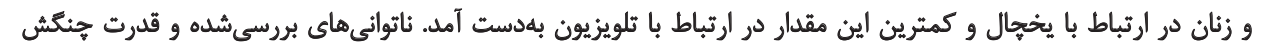

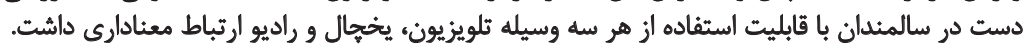

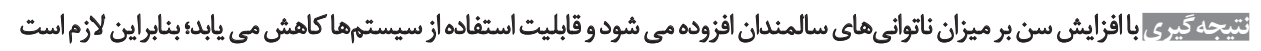

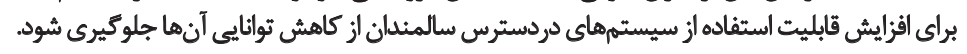

تاريخ دريافت: 19 تير هوبا

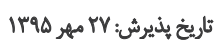

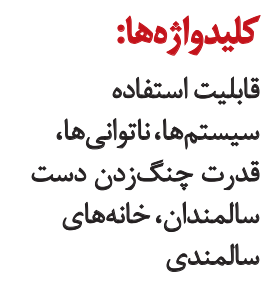

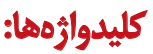

قابليت استفاده

سيستمها، ناتوانى هانها،

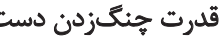

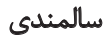

كاربريسند' مقبول شد [1] [بهطور كلى قابليت استفاده سيستمها

مقدمه - 20 - مقد

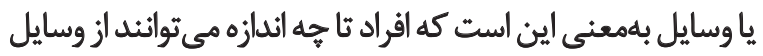

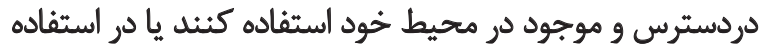

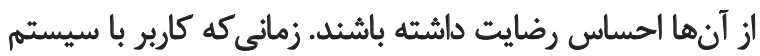

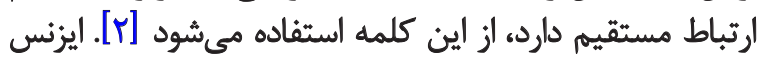

در اوايل دها •191 قابليت استفاده از سيستمها با تركيبي

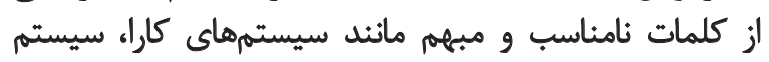

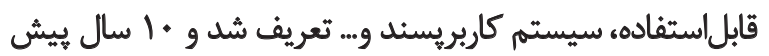
براى اولينبار كلمه قابليت استفاده بلعنوان جايكزين كلمه

1. User friendly

: نويسئده مسئول:

كتئر ناصر هاشمى نوراد

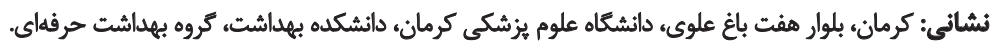

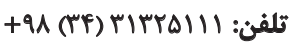

يست الكترونيكي: يلمن n_hasheminejad@kmu.ac.ir 


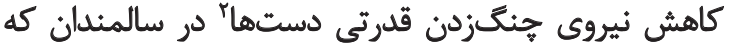

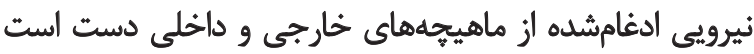

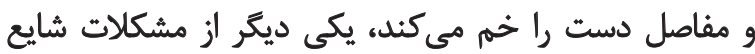

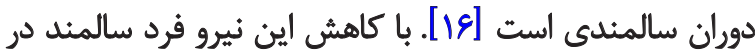

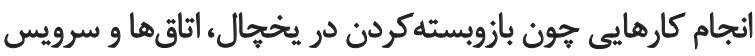

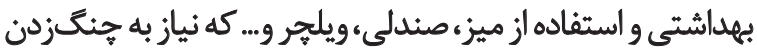

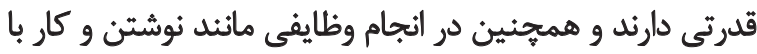

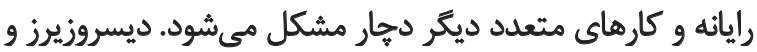

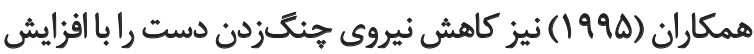

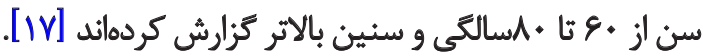
بهنظر ميرسد باوجود ناتوانىها و اختّلالات در سالمندان،

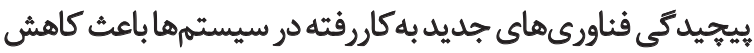

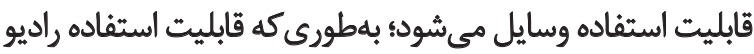

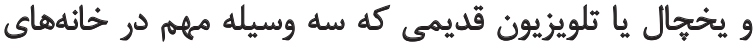

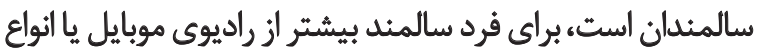
جديد سيستمهاى ديكًر است.

اكرجه استفاده از وسايلى مانند ثلويزيون و راديو و يخجال براى إناي

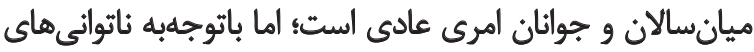

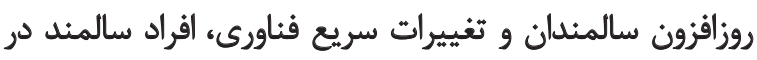

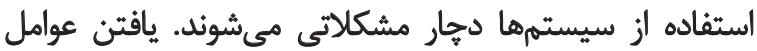

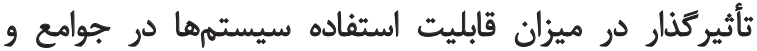

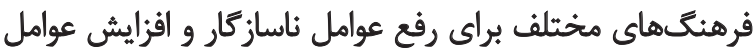

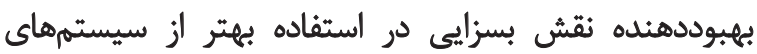
دردسترس بلويرثه در افراد سالمند دارد.

اين مطالعه با هدف بررسى ارتباط ناتوانىهاى بينايى، شنوايى،

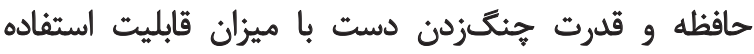

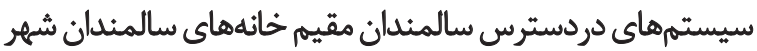

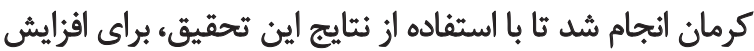

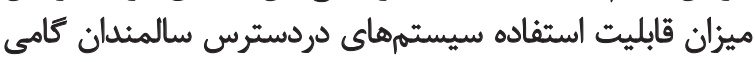

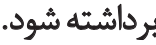

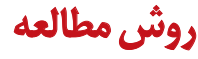

اين مطالعه از نوع مقطعى است كه بهصورت سرشمارى

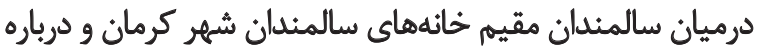
سه دستًاه مهم و دردسترس سالمندان (تلويزيون و ويخهال

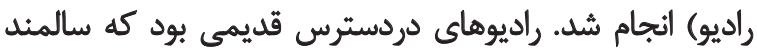

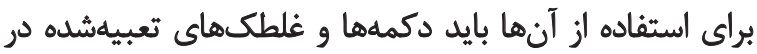

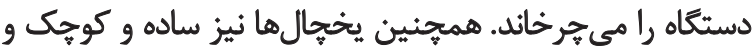

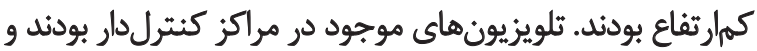

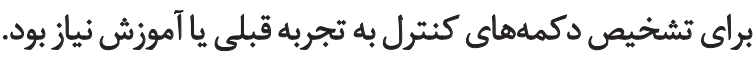
براى انجام يرُوهش اطلاعات با مراجعه حضورى به جهار مركز
(1991) قابليت استفاده را درجه توانايى كاربر باتوجهبه تجربهها

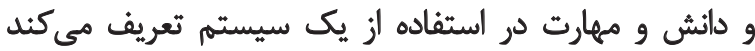

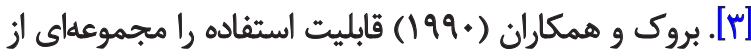

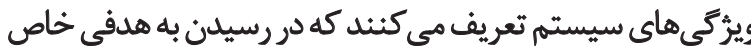

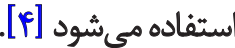

قابليت استفاده سيستمها با طراحى سيستمها ارتباط نزديكي إزيكي

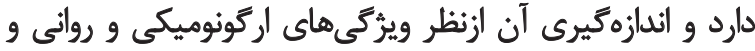

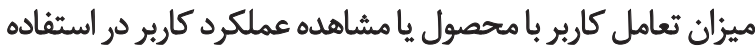

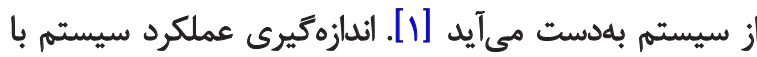

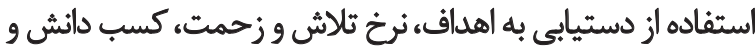

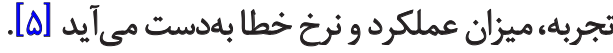

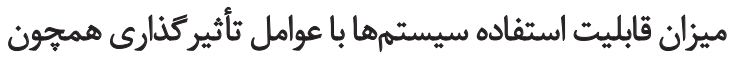

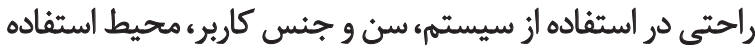

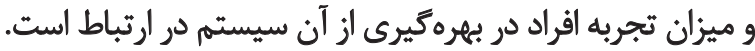

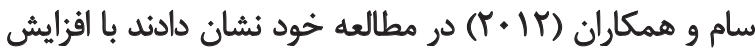

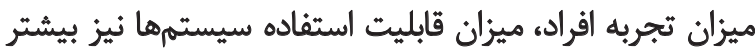

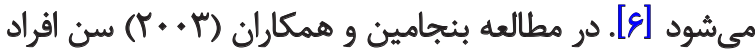

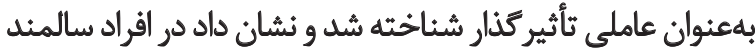

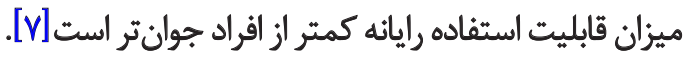

براساس تعريف سازمان بهداشت جهانى، افراد با سن •9 سال

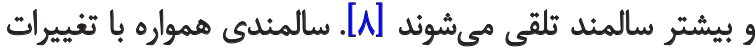

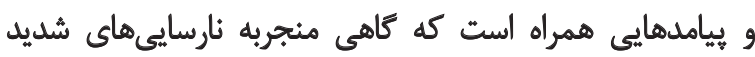

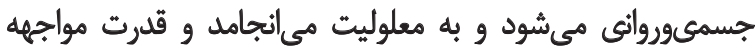

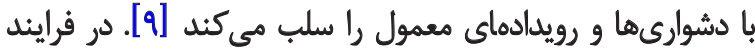

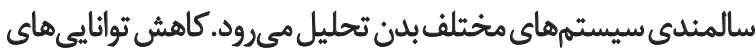

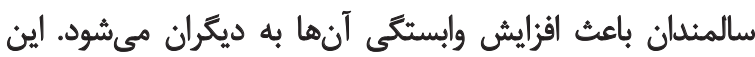

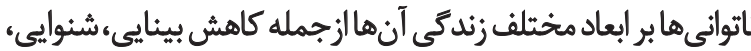

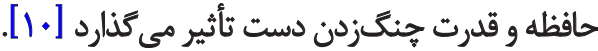

ناتوانى بينايى و كاهش شنوايى و ضعف در حافظيه مي تواند

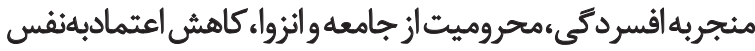

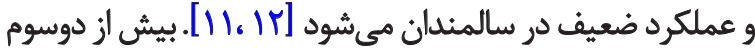

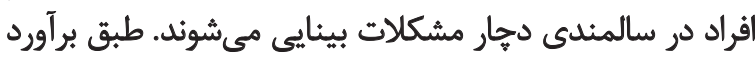

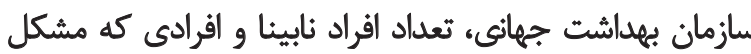

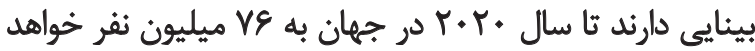

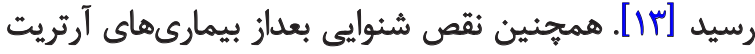

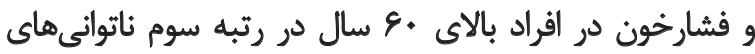

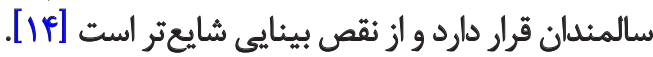
•ها تا •مدرصد سالمندان اختلالات حافظه دارند و بهدليل

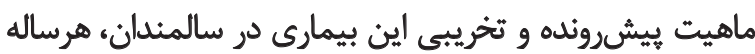

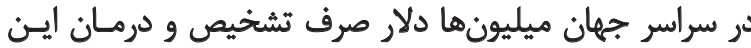

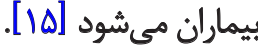


براساس دستورالعمل استاندارد ارائهشده توسط انجمن درمانكران

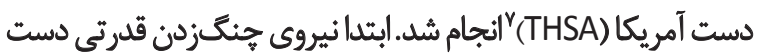

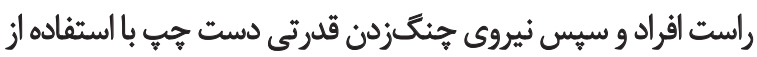

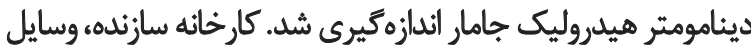

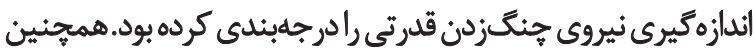

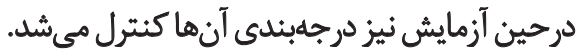

بلمنظور دستيابى به ميزان قابليت استفاده سيستمها (يخهال

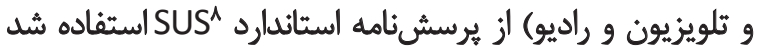

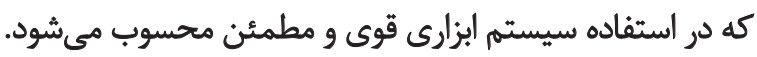

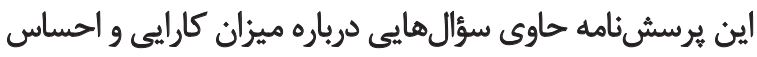

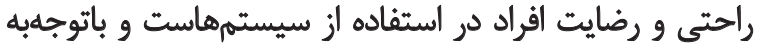

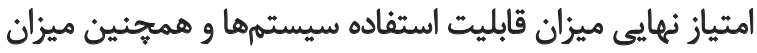

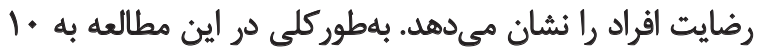

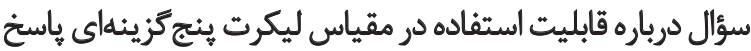

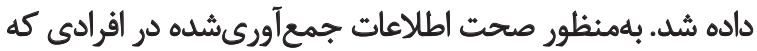

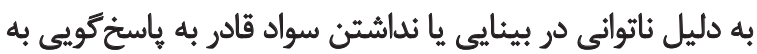

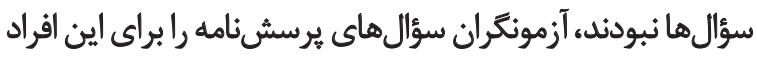
مي خواندند و در بركهماي مخصوص ثبت مي كردندان.

يس از جمعآورى اطلاعات آمار ثوصيفى و آمار تحليلى، از

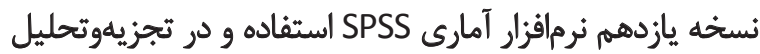

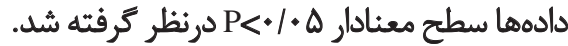

\section{يافتهها}

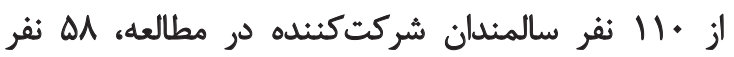

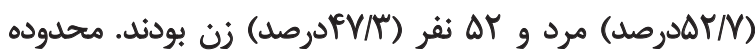

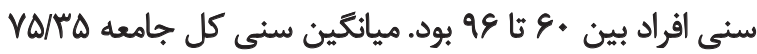

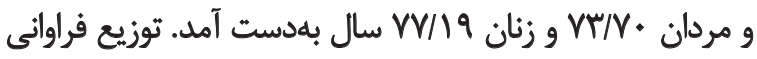

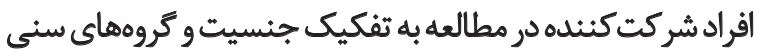

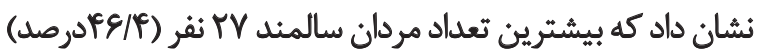

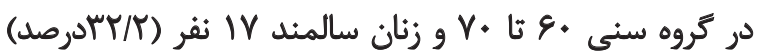

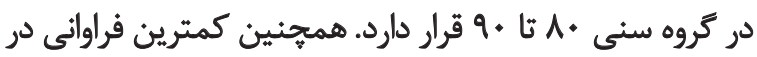

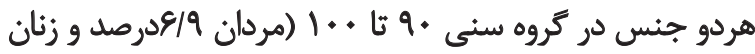
1/ آمرصد) است.

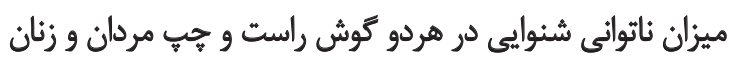

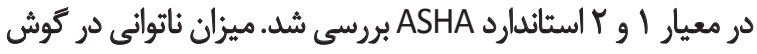

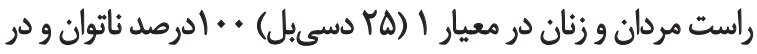

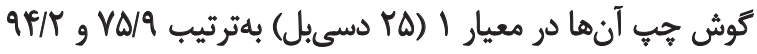

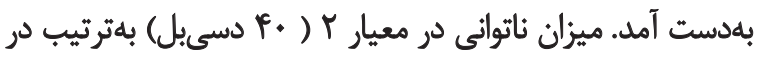

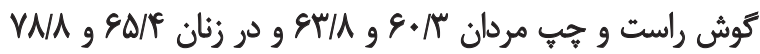

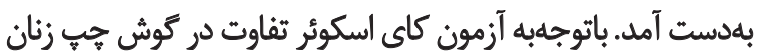

7. American Society of Hand Therapists

8. System Usability Scale

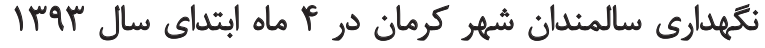

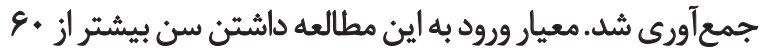

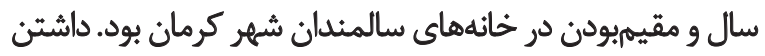

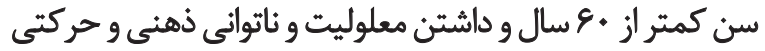

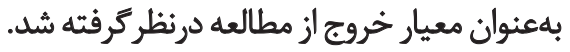

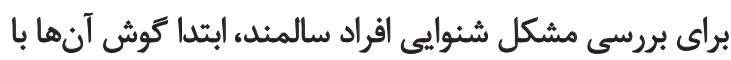

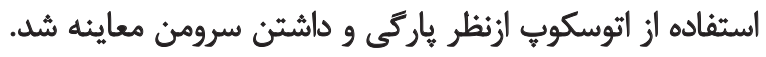

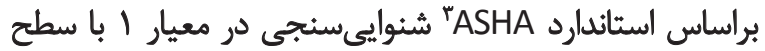

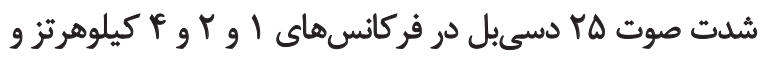

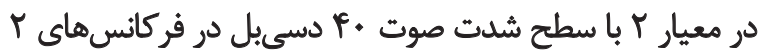

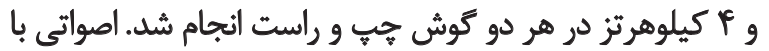

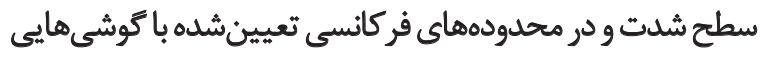

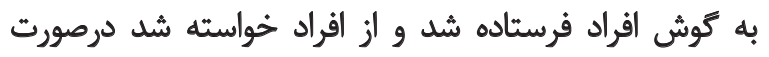

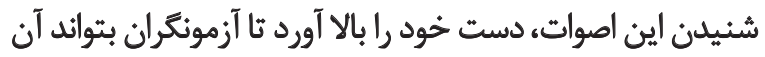
را بهعنوان توانايى شنوايع فرد ثبت كنيند.

قدرث بيناييى افراد باتوجلبه حدت بينايى (توانايى درك سيستم

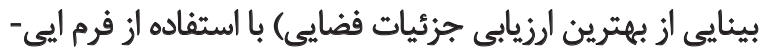

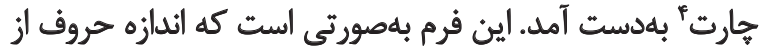

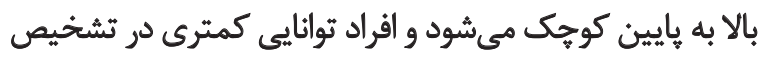

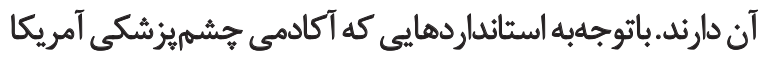

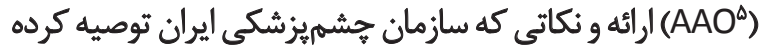

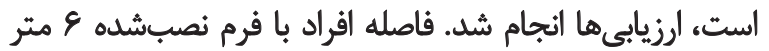

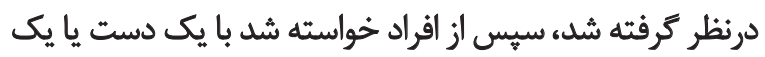

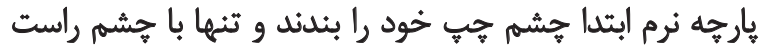

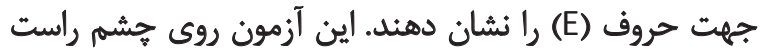

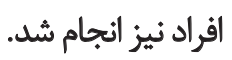

براى سنجش وضعيت شناختى و ناتوانى حافظه سالمندان از

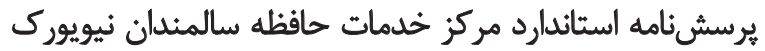

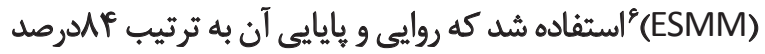

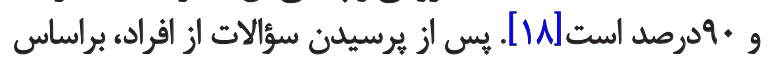

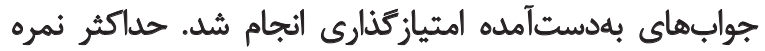

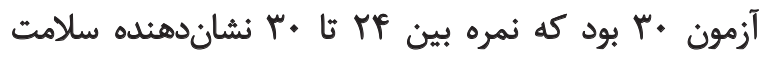

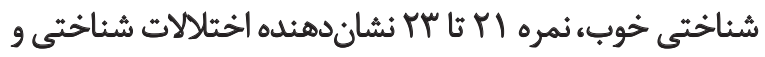

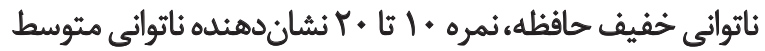

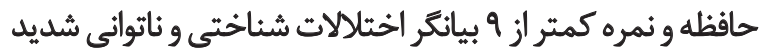
حافظه درنظركرفته شد. براى اندازميرى نيروى جِنَّزدن قدرتى دستها از دستَّاه

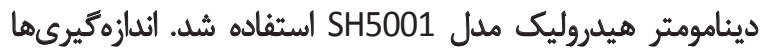

\footnotetext{
3. American Speech and Hearing Association

4. E chart

5. American Academy of Ophthalmology

6. Mini-Mental State Examination
} 
جدول ا. ميزان ناتوانى شئوايى، بينايى، سطوح حافظه، قدرت جنكّن و ميزان قابليت استفاده از سه وسيله تلويزيون و راديو و يخجال.

\begin{tabular}{|c|c|c|c|c|}
\hline P-value & مرود (تعداد) & مروحد (تعدان) & & \\
\hline- & $1 \cdots(\Delta T)$ & $1 \cdots(\Delta A)$ & 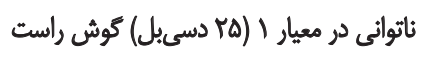 & \\
\hline.$* v$ & $9 \% / T($ (२q) & $\left.V \Delta / 9(9)^{\circ}\right)$ & 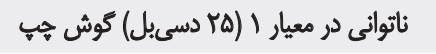 & \\
\hline .1 .96 & VNA(FI) & $\operatorname{RT} / A(T Y)$ & 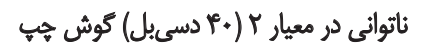 & \\
\hline.$/ 48$ & $g \Delta / P(M T)$ & $\varepsilon \cdot \pi(r \Delta)$ & ثاتوائى در هعيار Y (•P دسىبل) كوش راست & \\
\hline$\cdot / \pi$ & 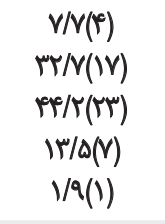 & 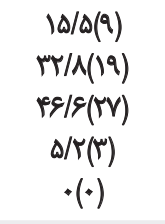 & 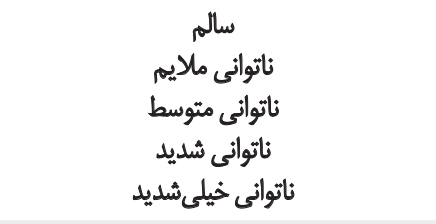 & بينائي جشم راست \\
\hline.$/ .4 r$ & $\begin{array}{c}r / A(Y) \\
I r / Q(Y) \\
P \varphi / T(Y Y) \\
r N A(Y+) \\
\cdot(\cdot)\end{array}$ & $\begin{array}{l}g / q\left({ }^{*}\right) \\
M I(M) \\
P I / Y(T Y) \\
I V / T(1+) \\
r / P(T)\end{array}$ & 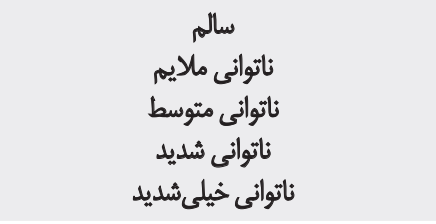 & يينائ جشمه هب \\
\hline+11 & $\begin{array}{c}1 / q(1) \\
q /(\Delta) \\
W D(f \&) \\
\cdot(\cdot)\end{array}$ & $\begin{array}{c}g / q(F) \\
r \cdot / N(T) \\
V T / F(T T) \\
\cdot(\cdot)\end{array}$ & مثفيفي & نمره نهائي قلدرت \\
\hline
\end{tabular}

(SD=1+/1)

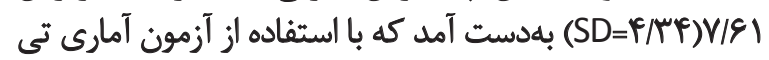

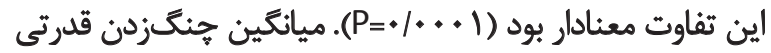

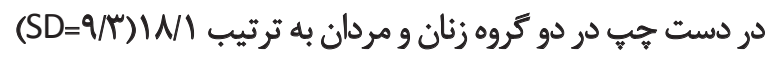

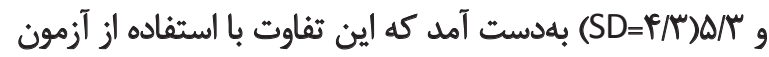

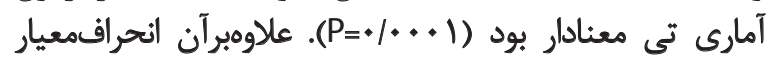

و مردان معنادار بود (جدول شماره ()). برايناساس تفاوت معنادارى

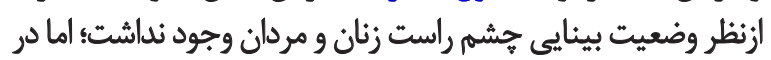

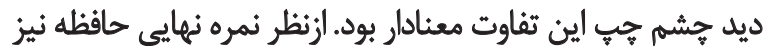
تففاوتى بين زئن و مردان مشاهده نشد.

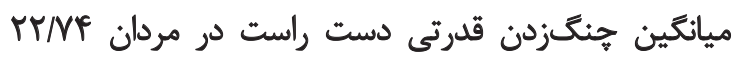

جدول r. ميانكين، انحرافمعيار، كميئه و بيشينه امتياز قابليت استفاده سه وسيله بررسىشده (تلويزيون و يخجال و راديو) در سالمئدان مطالعهشده به تفكيك جنسيت.

\begin{tabular}{|c|c|c|c|c|c|}
\hline كمترين & يبيشتين & أنحراف|سيثائدارد & ميائكين & & \\
\hline$\gamma+1$. & $M / \Delta$ & N. & $p q / p$ & قابليت استفاده از تلويزيون & \\
\hline$\Lambda . /$. & $F+1$ & $9 / 4$ & st/ & قابليت استفاده از يخجال & مردان (ه内 نفر) \\
\hline$\Lambda \Delta /$. & $\mathrm{W} / \mathrm{s}$ & 1.18 & $\Delta N / q$ & قابليت استفاده از راديو & \\
\hline$\gamma * 1$ & $M T / \Delta$ & NA & $r e / \mu$ & قابليت استفاده از تلويزيون & \\
\hline$V \otimes /$. & $r+\%$ & $8 / 9$ & $q \cdot / V$ & قابليت استفاده از يخهال & زنان (rه نفر) \\
\hline$\varepsilon \Delta /$. & $r \Delta /$ & $\mathrm{V} /$ & $0 . / 9$ & قابليت استفاده از راديو & \\
\hline$\pi / \Delta$ & v. & $N / F$ & ENI & قابليت استقاده از ثلويزيون & \\
\hline p. & A. & $N N^{*}$ & $81 / 1$ & قابليت استثاده از يخجهال & كل (•111 نْمر) \\
\hline ro & As & $9 / 9$ & $\Delta \Delta / 1$ & قابليت استفاده از راديو & \\
\hline
\end{tabular}




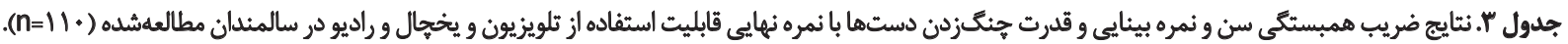

\begin{tabular}{|c|c|c|c|c|c|c|c|c|}
\hline قابز إديت استفاده & قابليت استثادهال & قابليت استفادئيون & 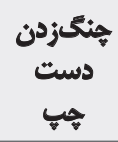 & دسثت راستىن & بينايى & رينايى & سن & \\
\hline & & & & & & & 1 & سن \\
\hline & & & & & & 1 & $-M^{\prime} q^{* *}$ & بينايع جشم رايست \\
\hline & & & & & 1 & + $/$ AVq & $-+11++*$ & بينايع جشم هيب \\
\hline & & & & 1 & . $18+1+4$ & $+/ \Delta A V^{* *}$ & $-+1 \Delta<q=$ & جنتش دست راست \\
\hline & & & 1 & ./9भाष" & $+18 \cdot 9^{* *}$ & $+/ \Delta W^{* *}$ & $-+/ \Delta A r^{* *}$ & جنتش دست جب \\
\hline & & 1 & $. / \Delta \mid f=*$ & $+/ F A V^{* *}$ & - $/ \Delta A r^{m}$ & $.1811^{4 * 0}$ & $-+18 W^{* *}$ & قابليت أستفاده تلويزيون \\
\hline & 1 & - Nar"** & -/AMT"** &.$/ \Delta T Q^{* *}$ & $.1910^{* *}$ & $\cdot / \Delta A r^{* * *}$ & $-+/ V 8+*$ & قابليت استفاده يخجال \\
\hline 1 &. $\mid R T^{*} \cdots$ & $.181 \mathrm{~N}^{\circ *}$ &. $\mid 8160 *$ & $.18 \cdot 9^{\circ * 0}$ & . / & ./AMnes* &.$- / \Delta W^{* *}$ & قابليت استفاده راديو \\
\hline
\end{tabular}

كمترين اين مقدار در ارتباط با تلويزيون (مردان

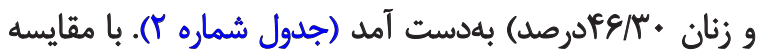

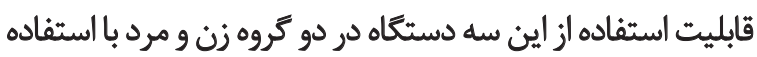

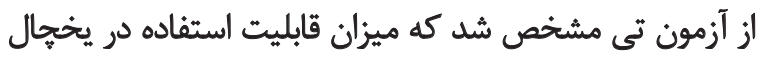

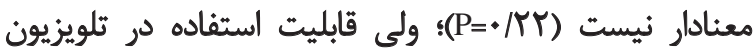

جنكَزدن قدرتي در هردو دست در كروه مردان بيشتر أز

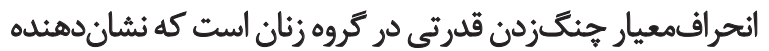
يراكندكي بيشتر در مردان مطالعهشيده است.

بيشترين ميزان قابليت استفاده در هردو كروه هردان و زنان

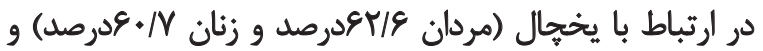

جدول †. نتايج آزمون لجستيك خطي براي تعديل و يافتن عوامل قابليت استفاده سه دستَّاه (تلويزيون و راديو و يخحال).

\begin{tabular}{|c|c|c|c|c|c|c|}
\hline \multirow{2}{*}{ Sig. } & \multirow{2}{*}{$\mathrm{T}$} & \multirow{2}{*}{$\begin{array}{c}\begin{array}{c}\text { Standardized } \\
\text { Coefficients }\end{array} \\
\text { Beta }\end{array}$} & \multicolumn{2}{|c|}{$\begin{array}{l}\text { Unstandardized } \\
\text { Coefficients }\end{array}$} & & \multirow{2}{*}{ Model } \\
\hline & & & Std. Error & B & & \\
\hline $.1 \%$ & IT/TMT & & g/NT & $V Q / \cdot \cdot r$ & (Constant) & \multirow{4}{*}{ SUS.TV } \\
\hline $.10+$ & $-\phi / 8+\mu$ & $-+10 \cdot 4$ & $+1+n$ & $-+/ 4+$ & Age & \\
\hline .1 .18 & rhera & $\cdot \pi \cdot V$ & $.1 \cdot n^{\infty}$ &.$/ \mathrm{VA}$ & Left & \\
\hline $.1 . \mathrm{YA}$ & T/MTA & .1184 & 1/TES & T/AT. & $\mathrm{m} 2 \mathrm{R}$ & \\
\hline .10 & $1 \cdot 14 \cdot 8$ & & NTVA & 19/Far & (Constant) & \multirow{6}{*}{ SUD. Refrigerator } \\
\hline .1. & $-8 / \mu \cdot 1$ & $-.18 \times 9$ & .1 .1 & $-\cdot 1 \Delta \cdot \Delta$ & Age & \\
\hline$\%$ & $r / \cdot r)$ & . RA. & VorV & P/809 & Sex & \\
\hline $.1 \cdot n$ & $-1 /$ AMP &.$- / 1199$ & plrar & -V/ATA & $O D$ & \\
\hline 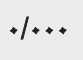 & $r(A V)$ & . / PAV & $.1+A V$ & . & Right & \\
\hline .1 .14 & r/pq. &.$/ 1 F \Delta$ & Wre & PIATA & $\mathrm{m} 2 \mathrm{R}$ & \\
\hline$\%$ & V/A1Q & & V/aqr & ER/FQQ & (Constant) & \multirow{5}{*}{ SUDS. Radio } \\
\hline $.1+\infty 1$ & -r/arV &.$- / r q u$ & $+1+v 9$ & $-+\pi r q$ & Age & \\
\hline .10. & f/rea &.$/ T V Q$ & $\% v e$ & $\cdot M$ & Right & \\
\hline | I I & MMAF & +/AAF & $\cdot \pi \cdot 1$ & . & Memory & \\
\hline $.10+t$ & $r / 9 \Delta)$ & $+\pi \cdot 1$ & $1 / 914$ & $\Delta / 8 \Delta 9$ & m1L & \\
\hline
\end{tabular}

닌 
مبنىبر نبود تفاوت در كاركرد بينايي بين زنان و مردان همسو

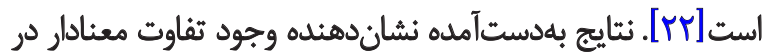

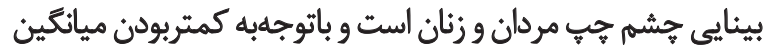

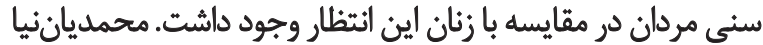

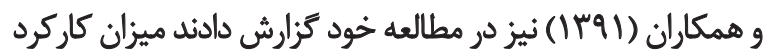

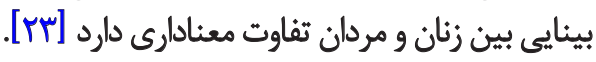

همجنين در اين يرؤهش (جدول شماره () تفاوت معنادارى

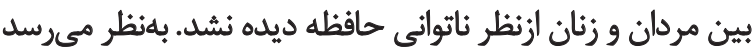

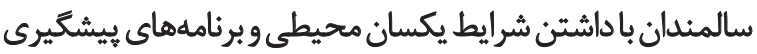

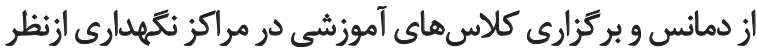

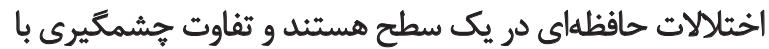

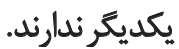

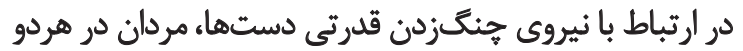

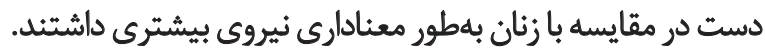

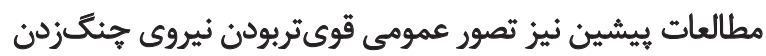

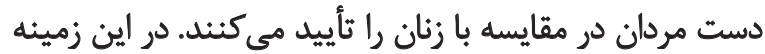

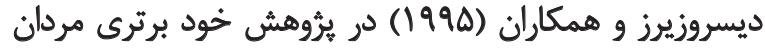

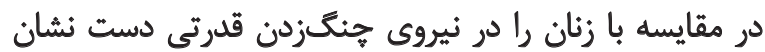

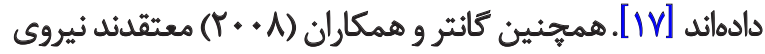

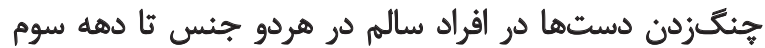

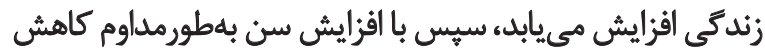

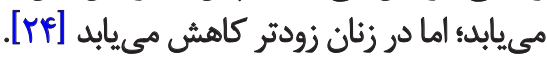

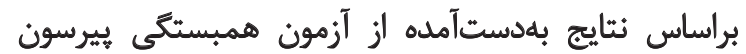

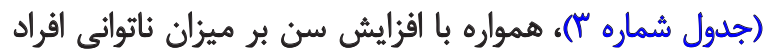

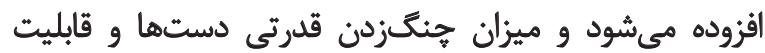

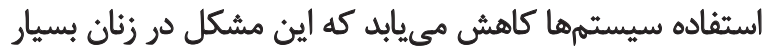

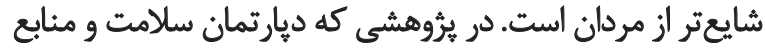

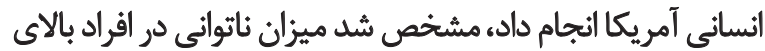

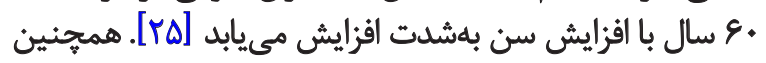

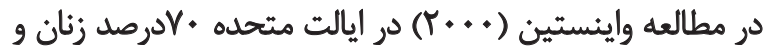

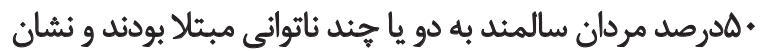

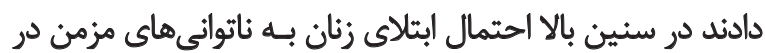

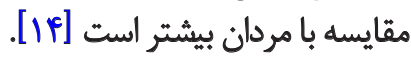

از بين سه دستكاه مهم و دردسترس سالمندان (تلويزيون

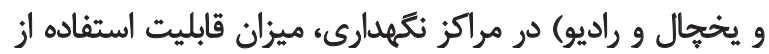

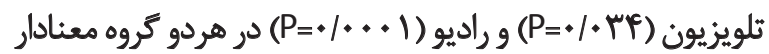

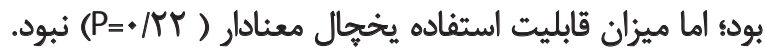

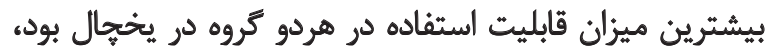

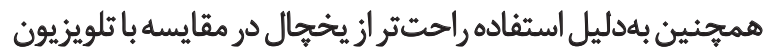

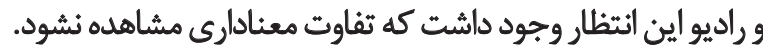

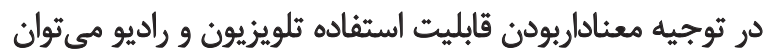

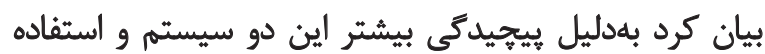

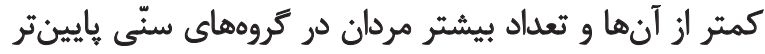

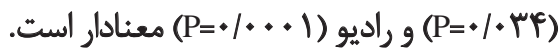

نتايج آزمون همبستگى يِيرسون نشان داد ارتباط بين سن و و

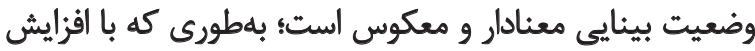

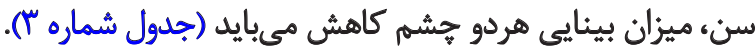

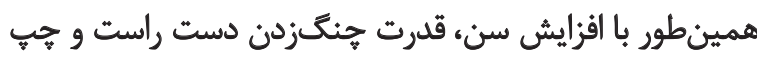

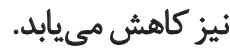

متغير سن با قابليت استفاده از هر سه وسيله بررسىشده

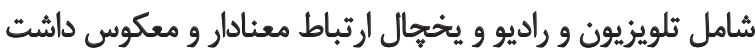

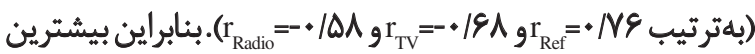

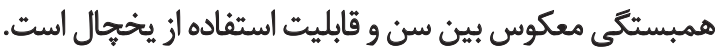
با افزايش سن، ميزان قابليت استفاده سيستمها در سالمندان

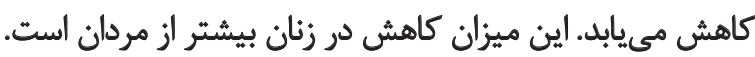

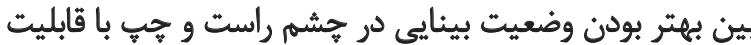

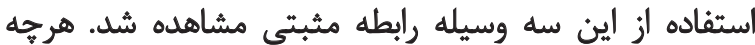

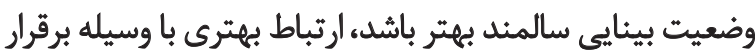

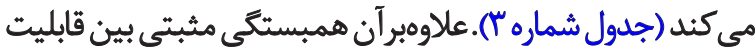

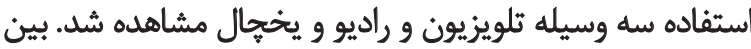

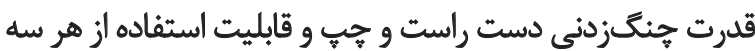

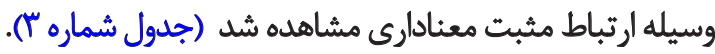
با انجام آزمون تى مشخص شد وضعيت شنوايى نيز با قابليت

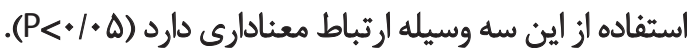

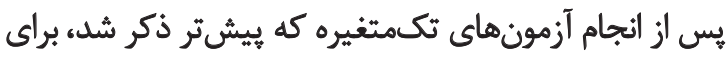

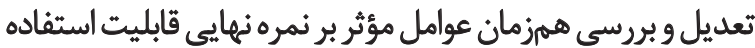

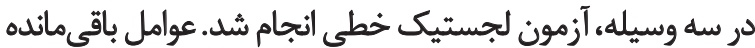

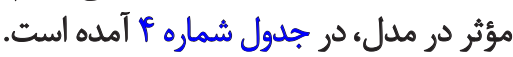

بحث

هدف از مطالعه حاضر بررسى ارتباط ناتوائىهاي شنوايى،

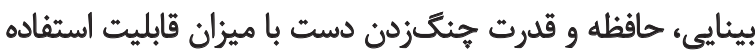

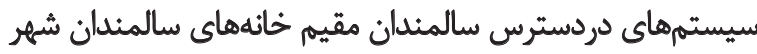

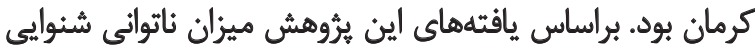

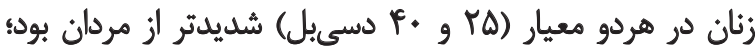

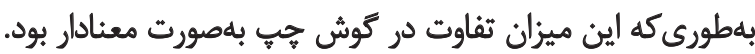

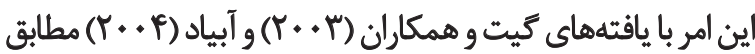

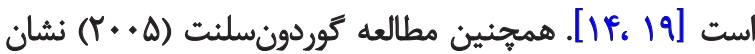

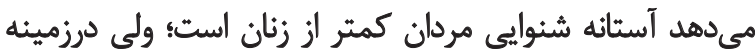

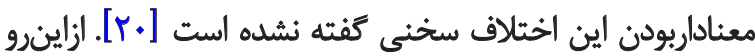

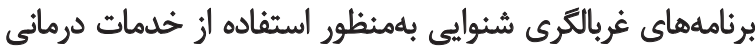

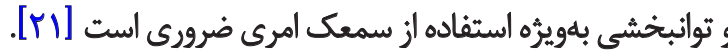

نتيجه بهدستآمده درزمينه بينايي افراد و نبود ثفاوت معناديار

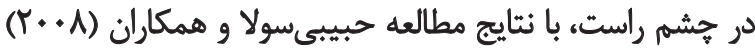


تلويزيون و راديو را آموزش دهند و در اين زمينه كلاسهايى بركزار

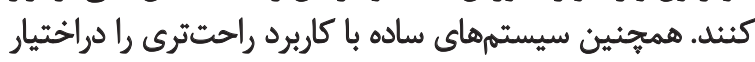

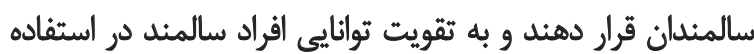

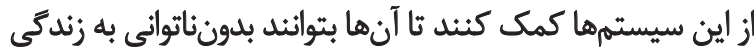

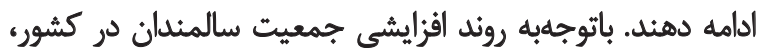

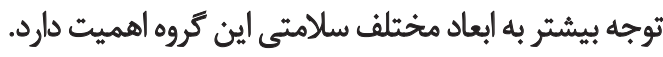

باكنون در ايران بهمنظور بررسي ميزان ارتباط ناتوانيىهاي

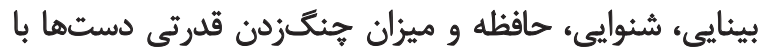

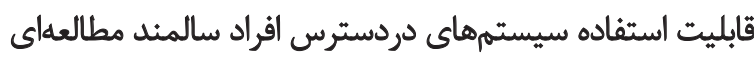

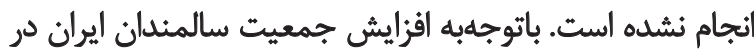

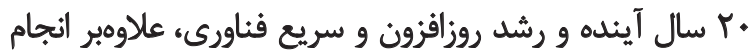

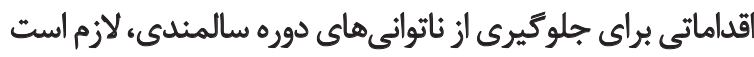

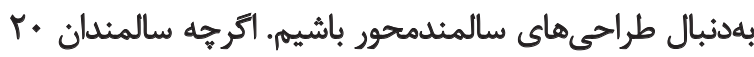

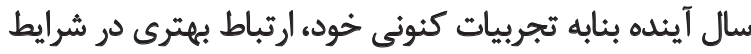

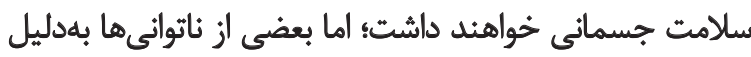
افزايش سن اجتنابنائذير است.

$$
\text { تشكر و قدردافي }
$$

بدينوسيله از آقاى منصورى، كارشناس شنوايىسنجى

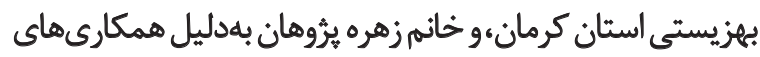

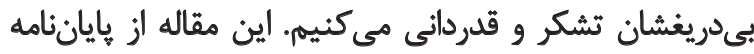

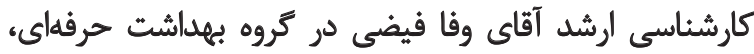
دانشكاه علوم يزشكى كرمان كرفته شده است
در مقايسه با زنان ميزان قابليت استفاده اين سيستمها در كروه

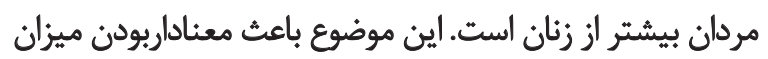

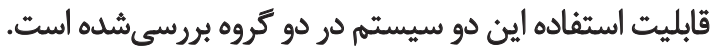

در ارتباط با كاهش ميزان قابليت استفاده سيستمها در مردان

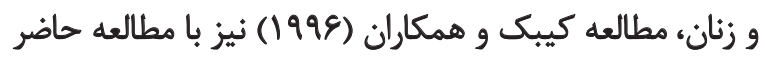

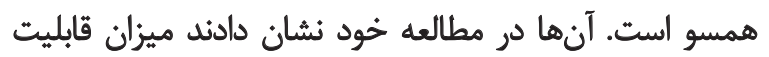

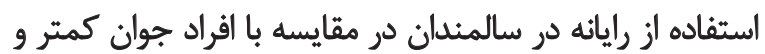

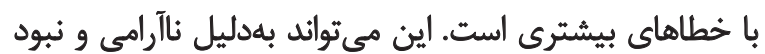

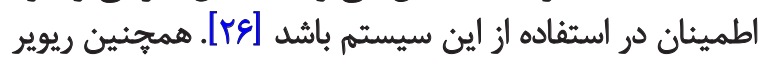

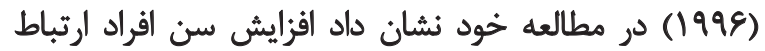

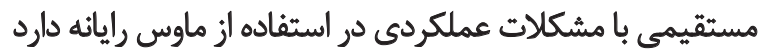

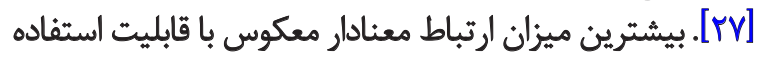

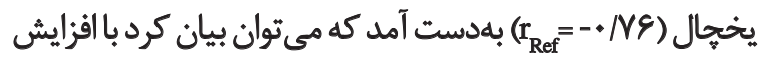
سن و كاهش اشتها تمايل به خوردوخوراك و قابليت استفاده از باز

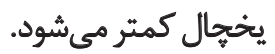

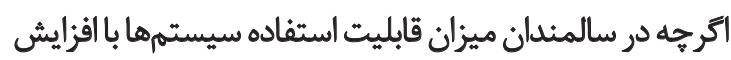

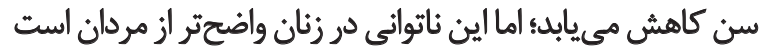

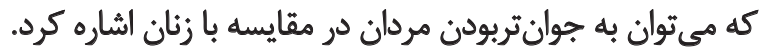

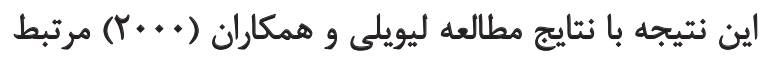

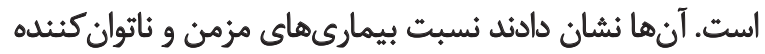

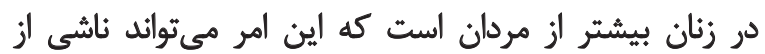

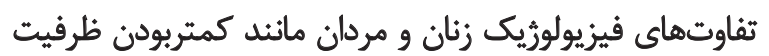

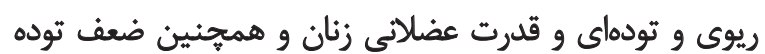
استخوانى زنان و تحليل سريعتر اين سيستمها دان در مقايسه بان

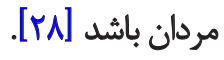

مسلم است كه با كاهش ميزان ناتوانىهاي فرده، كيفيت

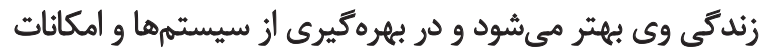

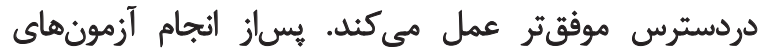

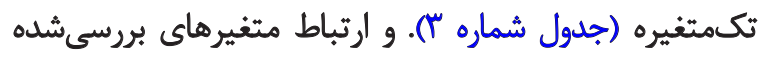

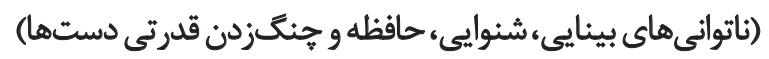

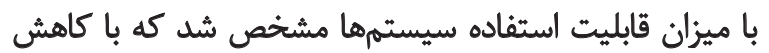

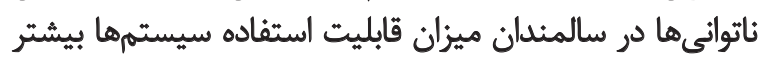

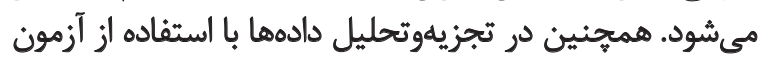

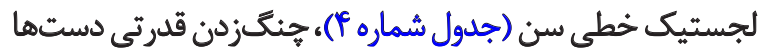

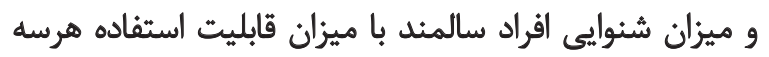
سيستم ارتباط معنادارى داشت.

\section{نتيجه كيرى نهايى}

جبلنظر ميرسد با افزايش روزافزون فناورى و توليد سيستمهاى

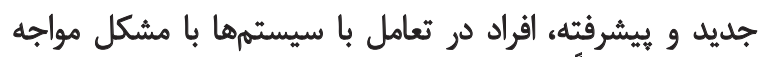

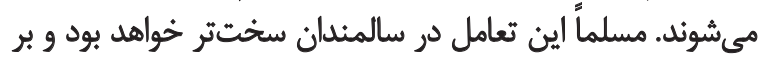

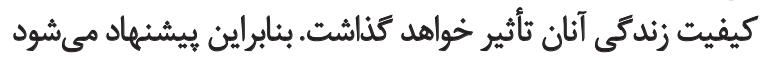

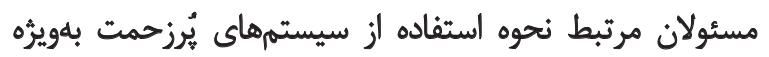




\section{References}

[1] Nigel B, Jurek K, Jonathan M. What is Usability? Paper presented at: The $4^{\text {th }}$ International Conference on HCI; 1991 Sep 1-6; Stuttgart, Germany.

[2] Brooke J. SUS-A quick and dirty usability scale. Smart Phone applications for people with Brain Injury. 2011. [Cited 09 Aug 2011]. Available from: B5_During_the_trial_usability_scale_ V1_09Aug11.pdf

[3] Eason K. Information technology and organisational change. Milton Park: Taylor and Francis; 1998.

[4] Brooke J, Bevan N, Brigham F, Harker S, Youmans D. Usability statements and standardisation-work in progress in ISO. In: Diaper D, editor. Human Computer Interaction-Interact' 90. Amsterdam: Elsevier; 1990

[5] Rengger N. Indicators of usability based on performance. In: Bullinger HJ, editor. Human Aspects in Computing: Design and Use of Interactive System with Terminals. Amsterdam: Elsevier; 1991.

[6] McLellan S, Muddimer A, Peres SC. The effect of experience on system usability scale ratings. Journal of Usability Studies. 2012; 7(2):56-67.

[7] Bederson BB, Lee B, Sherman RM, Herrnson PS, Niemi RG. Electronic voting system usability issues. Paper presented at: The SIGCHI Conference on Human Factors in Computing Systems. New York: Association for Computing Machinery; 2003.

[8] Zabihi A, Jafarianamiri SR, Aziznejad P, Hosseini SR, Bijani A. [Elderly Falling in houses and related factors (Persian)]. Journal of Babol University of Medical Sciences. 2013; 15(5):95-101.

[9] Sajadi H, Biglarian A. [Quality of life of eldery women in Kahrizak nursing house (Persian)]. Payesh. 2006; 6(2):105-58.

[10] Taghipour M, Hosseini SR, Kia K, Abbaspour M, Ghadimi R. [Prevalence of musculoskeletal pain and it's correlation to functional disability in elderly (Persian)]. Knowledge \& Health. 2013; $8(2): 76-82$

[11] Amini R, Kaldi A, Sahaf R, Haghani H, Hayatbakhsh R, Davatgaran K, Masoumi M. [Independency level in elderly blind war survivors: a cross sectional study (Persian)]. Iranian Journal of Ageing. 2012; 7(23):69-77.

[12] Clare L, Woods RT. Cognitive training and cognitive rehabilitation for people with early-stage Alzheimer's disease: A review. Neuropsychological Rehabilitation. 2004: 4(14):385-401. doi: 10.1080/09602010443000074

[13] Soori H, Javadi MA, Rafati N, Rabbanikhah Z, Delavari AR, Parsi Kia A. [Prevalence and causes of blindness and low vision in Tehran Province, 2005 (Persian)]. Journal of Ophthalmology (Bina). 2005; 11(2):151-63

[14] Weinstein BE. Geriatric audiology. $1^{\text {st }}$ ed. New York: Theime; 2000.

[15] Davison GC, Neale JM, Krince AN. Abnormal psychology. New York: John Wiley and Sons; 2004

[16] Mitsionis G, Pakos EE, Stafilas KS, Paschos N, Papakostas T, Beris AE. Normative data on hand grip strength in a Greek adult population. International Orthopaedics. 2009; 33(3):713-17. doi: 10.1007/s00264-008-0551-x
[17] Desrosiers J, Bravo G, Hebert R, Dutil E. Normative data for grip strength of elderly men and women. American Journal of Occupational Therapy. 1995; 49(7):637-44. doi: 10.5014/ajot.49.7.637

[18] Seyedian M, Falah M, Nouroozian M, Nejat S, Delavar A, Ghasemzadeh $\mathrm{H}$. [Preparing and validate the Persian version of the Mini-Mental State Examination (Persian)]. Journal of Medical Council of Iran. 2007; 25(4):408-14

[19] Abyad A. Screening for hearing loss in the elderly. Geriatrics Today. 2004; 7(2):43-45.

[20] Gordon-Salant S. Hearing loss and aging: New research findings and clinical implications. Journal of Rehabilitation Research and Development. 2005; 42(4):9-24. doi: 10.1682/jrrd.2005.01.0006

[21] Garstecki DC. Older adults: hearing handicap and hearing aid management. American Journal of Audiology. 1996; 5(3):25-34 doi: 10.1044/1059-0889.0503.25

[22] Habibi Sola A, Nikpour S, Sohbatzadeh R, Haghani H. [Quality of life in elderly people of West of Tehran (Persian)]. Iranian Journal of Nursing Research. 2008; 2(7):29-35.

[23] Mohamadyan Nia M, Foroughanm M, Rasafiani M, Hosseinzadeh S. [Evaluation of visual function and correlation with quality of life in elderly people of use Bushehr governmental clinics services (Persian)]. Iranian Journal of Ageing. 2012; 7(27):16-26.

[24] Gunther CM, Rickert M, Crispin A. Grip Strength in healthy Caucasion adult: Reference values. Journal of Hand Surgery. 2008; 33(4):558-66. doi: 10.1016/j.jhsa.2008.01.008.

[25] Department of Human Services. Disability among old people. United States: Department of Human Services; 1995.

[26] Kubeck JE, Delp ND, Haslett TK, McDaniel MA. Does jobrelated training performance decline with age? Psychology and Aging. 1996; 11(1):92-107. doi: 10.1037//0882-7974.11.1.92

[27] Riviere CN, Thakor NV. Effects of age and disability on track ing tasks with a computer mouse: Accuracy and linearity. Journal of Rehabilitation Research and Development. 1996; 33(1):6-16.

[28] Leveille SG, Resnick HE, Balfour J. Gender differences in disability: evidence and underlying reasons. Aging Clinical and Experimental Research. 2000; 12(2):106-12. doi: 10.1007/bf03339897 
